\title{
Driven lattice gas of dimers coupled to a bulk reservoir
}

\author{
Paolo Pierobon, ${ }^{1,2}$ Erwin Frey, ${ }^{1}$ and Thomas Franosch ${ }^{1,2}$ \\ ${ }^{1}$ Arnold Sommerfeld Center for Theoretical Physics (ASC) and Center for NanoScience (CeNS), Department of Physics, \\ Ludwig-Maximilians-Universität München, Theresienstr. 37, D-80333 München, Germany \\ ${ }^{2}$ Hahn-Meitner Institut, Abteilung Theorie, Glienicker Str. 100, D-14109 Berlin, Germany
}

(Received 7 April 2006; published 28 September 2006)

\begin{abstract}
We investigate the nonequilibrium steady state of a one-dimensional (1D) lattice gas of dimers. The dynamics is described by a totally asymmetric exclusion process (TASEP) supplemented by attachment and detachment processes, mimicking chemical equilibrium of the 1D driven transport with the bulk reservoir. The steady-state phase diagram and current and density profiles are calculated using both a refined mean-field theory and extensive stochastic simulations. As a consequence of the on-off kinetics, a phase coexistence region arises intervening between low and high density phases such that the discontinuous transition line of the TASEP splits into two continuous ones. The results of the mean-field theory and simulations are found to coincide. We show that the physical picture obtained in the corresponding lattice gas model with monomers is robust, in the sense that the phase diagram changes quantitatively, but the topology remains unaltered. The mechanism for phase separation is identified as generic for a wide class of driven 1D lattice gases.
\end{abstract}

DOI: 10.1103/PhysRevE.74.031920

PACS number(s): 87.10.+e, 02.50.Ey, 05.60.-k

\section{INTRODUCTION}

Driven lattice gas models have recently received much attention due to their possible application to intracellular transport [1]. The relevance of nonequilibrium transport to biology has been recognized for almost 40 years. In two pioneering papers MacDonald and co-workers $[2,3]$ modeled ribosome motion on mRNA using a one-dimensional (1D) driven lattice gas model, which later became known as the totally asymmetric simple exclusion process (TASEP).

In this model each particle occupies a site on a onedimensional lattice and advances stochastically unidirectionally (hence the term "totally asymmetric"). The nontrivial properties arise by imposing the constraint that each site may at most be occupied by one particle, and that moves are forbidden if the target site is already occupied (hence the term "exclusion"). Of particular interest are systems with open boundary conditions where particles may enter and leave the 1D lattice at the left or right boundary with rates $\alpha$ and $\beta$, respectively. Remarkably, this $1 \mathrm{D}$ driven system with open boundaries exhibits a nontrivial phase diagram with $\alpha$ and $\beta$ as control parameters [4].

For low extraction rate $\beta$ a high density (HD) phase occurs, where the density profile is controlled by the right boundary. Symmetrically, for low injection rate $\alpha$ a low density (LD) phase appears, where the density profile is determined by the left boundary. For high values of both $\alpha$ and $\beta$ the bulk density is independent of the boundary conditions and the system carries its maximal current (MC).

Exact solutions were found both for periodic and open boundary conditions through exact methods, such as recursive relations, Bethe and matrix product ansatz $[5,6]$.

The stochastic fluctuations have been investigated in terms of static and dynamic correlation functions by exact methods [7-9] and phenomenological approaches [10,11]. The rich phenomenology, as well as the availability of exact results has established TASEP as one of the paradigmatic models in nonequilibrium statistical mechanics [12-14].

Over many years the TASEP has served as a testing ground for a mathematical analysis of nonequilibrium prob- lems. More recently, the applications to biology have again become the focus of attention in the context of systems of extended particles. For example, ribosomes bind to mRNA occupying 12 codons and progressively advance by a single codon through two cycles of GTP (Guanosine-triphosphate) hydrolysis (elongation step). The implications of mutual steric hindrance of $\ell$-mers (monomers, dimers, 12-mers) have been studied by stochastic simulations and "refined mean-field theory" for $\ell$-TASEP $[15,16]$. The picture already found for monomers has been corroborated also by exact methods (Bethe ansatz [17] and mapping to zero range process [18]).

Intracellular transport, where a molecular motor (kinesin) is moving along a molecular track (microtubule), is yet another example of 1D nonequilibrium transport in a biological system (see, e.g., Refs. $[19,20]$ ). In marked contrast to directed motion of ribosomes on mRNA here there is an exchange of molecular motors with the surrounding cytosol acting as a particle reservoir. This observation has motivated a recently proposed extension of TASEP, by supplementing the unidirectional hopping with Langmuir (on-off) kinetics $[21,22]$. Single motor experiments suggest that both processes compete on the scale of the microtubule, i.e., a motor explores a significant fraction of the track before detaching [23]. To capture such an interplay mathematically, a mesoscopic limit has been suggested, where local adsorptiondesorption rates have been rescaled in the limit of large but finite systems, such that the gross rates are comparable to the injection-extraction rates at the boundaries [22]. The study of this system has shown an unexpectedly rich and different phase diagram with phase coexistence regions. Surprisingly in the mesoscopic limit the density profiles show a sharp discontinuity (a shock separating low from high density phases) or cusp in a large portion of the phase diagram.

Here we propose a model for intracellular transport incorporating both coupling to a reservoir as well as the finite extension of particles. The model is motivated by the fact that many processive molecular motors (e.g., kinesins, dyneins, and myosin V) are composed of two heads (trail and lead) that bind specifically each to a subunit of the molecular 
track ( $\alpha$ - and $\beta$-tubulin in the case of microtubules). Hence we concentrate on particles which occupy two sites, i.e., dimers, and advance by a single site per hopping event.

The theoretical challenge arises from finding an appropriate and quantitative description that captures both the on-off kinetics as well as the $\ell$-TASEP features on an equal footing. Whereas for monomers a straightforward decoupling of correlations yields a consistent mean-field theory, such an approach fails even to describe the chemical equilibrium of a pure on-off kinetics [15]. In the following paragraphs we review some aspects of our model where the geometric constraints usually play an important role introducing nontrivial static and dynamic correlations.

At the static level the geometrical frustration emerges already in the equilibrium thermodynamics of the onedimensional Tonks gas. Identical extended particles are distributed according to the grand canonical ensemble on a onedimensional lattice. A hard core repulsion reduces the task to a combinatorial problem $[24,25]$. It turns out that the single site occupation densities do not determine the configurational probabilities, contrary to the monomer case. There the Langmuir kinetics are specified in terms of attachment and detachment rates $\omega_{A}$ and $\omega_{D}$, or equivalently the binding constant $K=\omega_{A} / \omega_{D}$. The coverage density is simply determined by a single site consideration, resulting in the Langmuir isotherm: $\rho_{I}=K /(K+1)=\omega_{A} /\left(\omega_{A}+\omega_{D}\right)$. This is to be contrasted with the dimer case where a full evaluation of the grand canonical partition function is required leading to an equilibrium coverage density [26]

$$
\rho_{I}=1-\frac{1}{\sqrt{1+4 K}} .
$$

At a dynamical level a nontrivial approach to the steady state is found in the on-off kinetics in the fast attachment limit: at short time scales only deposition processes are frequent, while detachment processes are still unlikely. The kinetics belong to the class of problems referred to as random sequential adsorption (RSA) and the so-called "Flory plateau" [27] is reached exponentially fast. After this transient the particles can detach (on longer time scales) freeing new gaps; this process results in a long tail relaxation where the isotherm is approached with a power law $t^{-1 / 2}$. Such behavior has been explained using the mapping of the gap dynamics to a $1 \mathrm{D}$ reaction diffusion system $A+A \rightarrow \varnothing$ (for a review see Refs. [28-30] and references therein) for which this anomalous power law dynamics is known. This two step relaxation has been found also in other models like in irreversible deposition of dimers (i.e., RSA) with diffusion [31] and is intrinsically related to the extended nature of the particles.

Driving a system of extended particles far from equilibrium, e.g., introducing the $\ell$-TASEP dynamics, suggests a variety of competing time scales and ordering phenomena, i.e., nonequilibrium phase transitions. The purpose of this paper is to shed light on density and current profiles in the stationary state for a model incorporating such aspects, as well as to provide a complete analytical theory supported by simulated data.

This paper is organized as follows: in Sec. II we specify the model under consideration and introduce the used for-

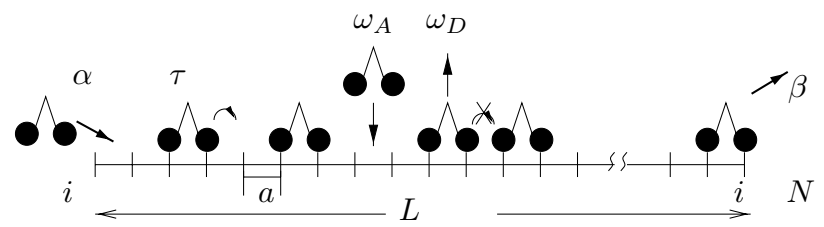

FIG. 1. Schematic representation of the model and allowed moves: forward jump (with rate $\tau=1$ ), entrance at the left boundary (with rate $\alpha$ ), exit at the right boundary (with rate $\beta$ ), attachment (with rate $\omega_{A}$ ), and detachment (with rate $\omega_{D}$ ) in the bulk.

malism. Section III exemplifies results of the stochastic simulations and puts extracted features into proper perspective. In Sec. IV we construct a suitable set of mean-field equations relying on results for $\ell$-TASEP and on-off kinetics. In Sec. V, with the help of these equations, we rationalize the MC data and study the complete phase diagram. In the Conclusion, Sec. VI, we discuss the robustness of the model, give a possible generalization to $\ell$-mers, and summarize our main results.

\section{MODEL AND NOTATION}

We consider a finite one-dimensional lattice with sites labeled $i=1, \ldots, N$, of unit length $L=1$; consequently the lattice spacing reads $a=L / N$. The first two sites $(i=1,2)$ and the last two sites, $(i=N-1, N)$ represent, respectively, the left and right boundary. The lattice is partially covered by dimers, i.e., composite objects consisting of two monomers rigidly tied together. A dimer occupies two lattice sites, see Fig. 1; we refer to the monomer to the right of the dimer as the lead head and correspondingly to the left monomer as the trail head.

A microscopic state $\mathcal{C}$ of the system consists of a configuration of nonoverlapping indistinguishable dimers on the lattice. Nonoverlapping requires that no two heads (trail or lead) occupy the same lattice site. We specify the dynamical evolution by the following set of updating rules:

(i) If the lead head of a dimer occupies a site $i$ $=2, \ldots, N-1$ and the following site is empty, the dimer advances one step with unit rate.

(ii) At site $i=1$ a dimer enters the lattice with its trail head with rate $\alpha$ provided that the first two sites are empty.

(iii) A dimer with its lead head on site $i=N$ leaves the lattice with rate $\beta$ emptying the last two sites.

(iv) Everywhere in the bulk (i.e., trail head on a site $i$ $=2, \ldots, N-2)$ a dimer leaves the lattice emptying two sites with a site independent detachment rate $\omega_{D}$.

(v) Everywhere in the bulk $(i=2, \ldots, N-2)$ a dimer enters the lattice with its trail head, provided that the considered site as well as its right neighbor is empty, with a site-independent attachment rate $\omega_{A}$.

The first three rules encode the usual totally asymmetric simple exclusion process of extended objects ( $\ell$-TASEP), while the last two implement the coupling of the lattice to a reservoir of dimers with fixed chemical potential [32].

The rules entail that a complete description of the underlying stochastic Markov process is given in terms of the 
time-dependent probability $P(\mathcal{C}, t)$. The time evolution is governed by the associated master equation

$$
\partial_{t} P(\mathcal{C})=\sum_{\mathcal{C}^{\prime} \neq \mathcal{C}}\left[W_{\mathcal{C}^{\prime} \rightarrow \mathcal{C}} P\left(\mathcal{C}^{\prime}, t\right)-W_{\mathcal{C} \rightarrow \mathcal{C}^{\prime}} P(\mathcal{C}, t)\right],
$$

where the transition rates $W_{\mathcal{C}^{\prime} \rightarrow \mathcal{C}}$ can be inferred form the dynamical rules.

It is expected that the system evolves towards a stationary ensemble $P^{s t}(\mathcal{C})$ for long times. Due to the lack of detailed balance this macroscopically stationary state does not correspond to an equilibrium ensemble, i.e., there is no GibbsBoltzmann measure to be inferred from thermodynamics arguments. The macroscopic quantities we aim to compute are understood as averages over this distribution.

To exploit probabilistic methods we label sites according to: empty (state $s=0$ ), occupied with the trail head (state $s$ $=1$ ) or with the lead head (state $s=2$ ). The configuration $\mathcal{C}$ can be represented as a string of occupation number $\mathcal{C}$ $=\left\{n_{1}, \ldots, n_{N}\right\}$, with $n_{i} \in\{0,1,2\}$. The quantity of fundamental interest in the following is the average site-dependent dimer density in the stationary state. In particular we shall derive equations for the density of the lead head $\left(n_{i}=2\right)$ at site $i, \rho_{i} \equiv\left\langle\delta_{n_{i}, 2}\right\rangle$. Since the dimers are rigidly connected, the corresponding density for the trail head at site $i$ simply reads $\rho_{i+1}$.

The probability of having site $i$ in state $s$ will be denoted by $p(i, s) \equiv P\left(n_{i}=s\right)$. Since the states mutually exclude, the probability $p(i, 2)$ immediately yields the average lead head density: $\rho_{i}=p(i, 2)$. The coverage density $\rho_{i}^{c}$ is the sum of the lead head density and the trail head density, i.e., $\rho_{i}^{c}=p(i, 2)$ $+p(i+1,2)$. Consistently with the notation the joint probability will read $p\left(i, s ; j, s^{\prime}\right)$. We also shall need the conditional probability $p\left(i+1, s \mid i, s^{\prime}\right)$, i.e., the probability for site $i+1$ to be in state $s$ provided site $i$ is in state $s^{\prime}$.

In the simulations we will concentrate on the average coverage density $\rho_{i}^{c}$, and the spatially resolved current $j_{i}$, defined as the flux of particles through site $i$ per unit time. Only averages on the stationary state will be considered: a comparison between ensemble and time-moving averages corroborates the hypothesis that the system is ergodic.

As indicated in the Introduction, a nontrivial competition between boundary induced phenomena and bulk dynamics arises in the mesoscopic limit, i.e., the gross on and off rates, $\Omega_{A}$ and $\Omega_{D}$, should be of the same order of magnitude as the boundary entry and exit rates $\alpha$ and $\beta$. This requires that the single site on and off rates, $\omega_{A}, \omega_{D}$, scale with the system size $N$ as

$$
\omega_{A}=\frac{\Omega_{A}}{N}, \quad \omega_{D}=\frac{\Omega_{D}}{N}
$$

with $\Omega_{A}$ and $\Omega_{D}$ fixed.

\section{MC RESULTS AND DESCRIPTION OF THE PHASE DIAGRAM IN GENERAL}

In this section we present the method used to perform numerical simulations and the results obtained from them.
Density profiles much different from the TASEP motivate our interest in the model.

\section{A. Method}

We have simulated the stochastic equation through a kinetic Monte Carlo algorithm, to determine the average density profile in the stationary state with high accuracy. The results have been obtained using a random sequential updating algorithm by Bortz, Kalos, and Lebowitz (BKL method or $n$-fold way) [33,34]: a list of all sites which are possible candidates for a successful move is stored and dynamically updated. The method is (for the present case) faster than the conventional algorithms and constitutes a reliable way to simulate real time dynamics, although here we focus on equal-time averages in the stationary state (note that the algorithm is equivalent to Gillespie's, commonly used in chemical reactions [35]).

Explicitly, we keep three lists of sites for the respective allowed moves: there are $N_{J}$ sites where particles can perform a jump forward, $N_{A}$ sites that may accept a particle from the reservoir, and $N_{D}$ sites from which a particle can detach. Furthermore, we keep track of the occupation of the boundaries $\left(n_{0}\right.$ and $\left.n_{N}\right)$. One of these moves is selected randomly with the appropriate weights: $1 \times N_{J}, \omega_{A} \times N_{A}, \omega_{D}$ $\times N_{D}, \alpha \times\left(1-n_{1}\right), \beta \times n_{N-1}$. Then we increment time by an interval drawn from an exponential distribution with time scale $T=\left[\alpha\left(1-n_{0}\right)+\beta n_{N}+N_{J}+\omega_{A} N_{A}+\omega_{D} N_{D}\right]^{-1}$. The lists are updated correspondingly and the procedure is iterated for many events. We have started the algorithm from an empty configuration and after the stationary state has been reached, the coverage density and the current have been measured. Moving time averages typically cover a window of $O\left(10^{6}\right)$ time units. We have investigated finite-size effects by considering lattices varying from 128 up to 4096 sites. Furthermore, we have checked the ergodicity by comparing timemoving and ensemble averages. The longest simulations performed took approximately a day on a $1-\mathrm{GHz}$ processor.

\section{B. Results}

We exemplify our results by fixing the binding constant $\left(K=\Omega_{A} / \Omega_{D}\right)$ to $K=3$ and the entrance and exit rates to $\alpha$ $=0.1$ and $\beta=0.6$; see Fig. 2 . The parameters chosen here are to illustrate the phase behavior; biologically relevant parameters are discussed for monomers in Ref. [36] and a faithful mapping to experimental knobs is presented in Ref. [37].

We have measured the stationary density profiles as well as the current for different values of the detachment rate $\Omega_{D}$. For very small $\Omega_{D}$ we find almost flat profiles for both $\rho$ and $j$, in accordance with the picture of simple dimer TASEP $[3,15,16]$. On the other hand, for very large detachment rates the on and off kinetics dominates the bulk of the density profile. However, contrary to equilibrium thermodynamics of on and off kinetics, a large directed current is simultaneously present.

For intermediate values of the detachment rate, neither the density profile nor the current is constant anymore. There appear rather narrow regions where the density steeply rises 

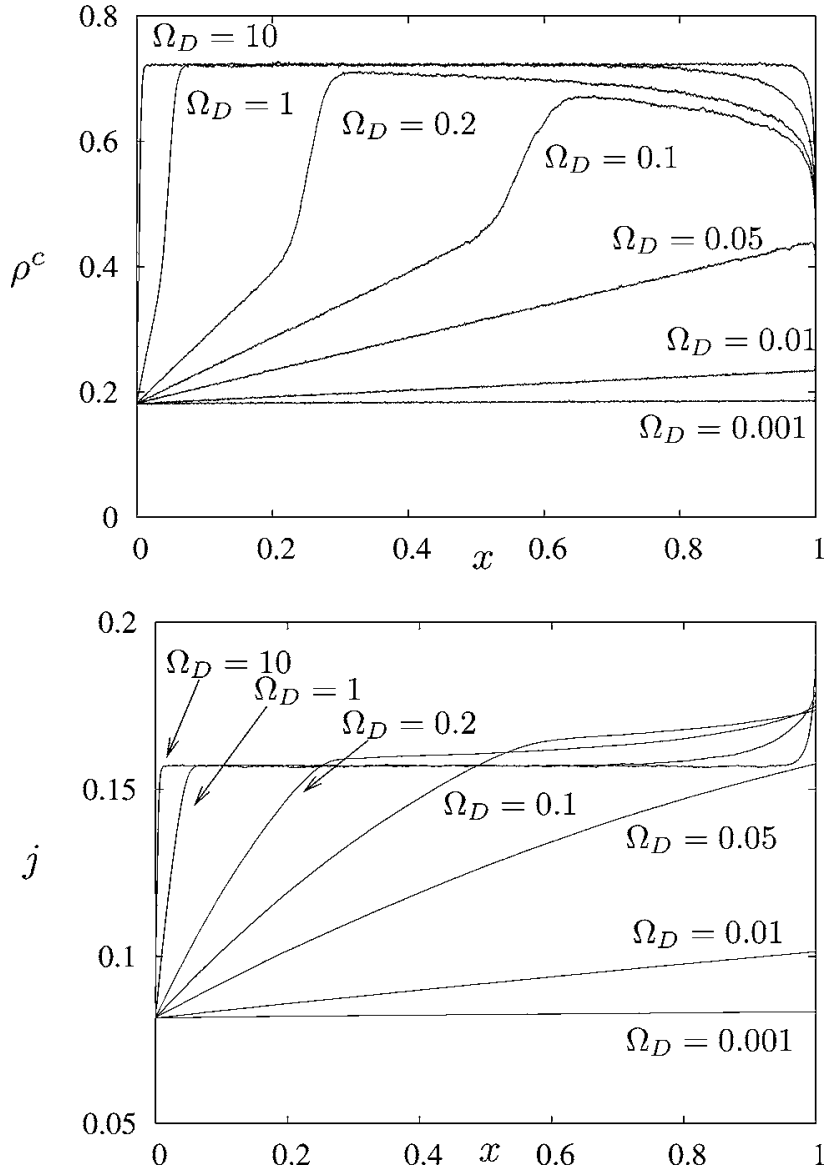

FIG. 2. Stochastic simulations results for stationary density profiles and corresponding currents for a system of $N=4096$ lattice sites. The $x$ axis represents the normalized position, i.e., $x=i / N$. Parameters have been fixed to $\alpha=0.1, \beta=0.6, K=3$, and $\Omega_{D}$ are varied as indicated in the figures.

from an almost linear profile to one that approaches the isotherm value. Such findings are similar to the ones of monomeric TASEP coupled to Langmuir kinetics [22,41], where it has been shown that the steep increase corresponds to a domain wall of a coexistence phase in the mesoscopic limit.

\section{CONSTRUCTION OF THE MEAN-FIELD EQUATION}

Since the stationary density profiles for TASEP of monomers coupled to Langmuir kinetics have been successfully described in terms of mean-field theory, it appears promising to look for an analytical description for dimers also. However, it turns out that in the case of dimers the conventional mean-field approach, i.e., a decoupling of correlations at lowest order starting from the quantum Hamiltonian formulation, see, e.g., Ref. [41], is inappropriate: the nontrivial correlations, arising from the fact that the particles are extended, manifest themselves already at the level of pure TASEP of dimers [15].

The failure of conventional mean-field approach requires the introduction of phenomenological methods. The aim of this section is to identify useful strategies that are capable to deal first with the simple on and off kinetics of extended objects without directed motion and second with pure TASEP for dimers. We will then combine the two cases to obtain a formulation for the complete problem, and we will provide arguments that the aspects of correlations are captured on a reliable level for both processes. In order to capture the competition between boundary effects (injection and extraction) and bulk processes (attachment and detachment) in a finite system, the mean-field approach has to properly balance the relative importance of both mechanisms. To emphasize this aspect we shall refer to our analytical approach as a mesoscopic limit.

The density profile in the mesoscopic limit will be described in terms of a balance equation. The evolution of the course-grained dimer density is determined by (i) a flux $j$ of dimers within the lattice that encodes the asymmetric diffusion, (ii) two source terms that represent the on-off kinetics of dimers:

$$
\partial_{t} \rho=-\partial_{x} j+F_{A}-F_{D}
$$

The nontrivial task is to provide reasonable expressions for the current and the sources in terms of the local density. In Sec. V we shall compare the analytical results to stochastic simulations and demonstrate the validity of this approach.

\section{A. On-off kinetics of dimers}

\section{Probabilistic approach}

The dynamics of the on-off kinetics in a rate equation approach is described by the difference between a gain term $F_{i}^{A}$ and a loss term $F_{i}^{D}$ :

$$
\partial_{t} \rho_{i}=F_{i}^{A}-F_{i}^{D}
$$

These two terms are proportional to the attachment and detachment rates $\omega_{A}$ and $\omega_{D}$. Clearly the detachment flux is exactly given by $F_{i}^{D}=\omega_{D} \rho_{i}[38]$.

An attachment event at site $i$ (lead head) requires both sites $i$ and $i-1$ to be empty (i.e., in state 0$)$. The corresponding attachment flux then reads $F_{i}^{A}=\omega_{A} p(i-1,0 ; i, 0)$, and knowledge of the joint probability is needed. In order to obtain closed equations for the lead head density we break the hierarchy of joint probabilities resulting from the master equation. Here, we rely on an ad hoc approximation for the conditional probabilities: $p(i, 0 \mid i-1,0) \simeq p[i, 0 \mid(i-1,0) \vee(i$ $-1,2)$ ] [39]. The idea is that the probability of having site $i$ empty is independent of having a lead head or a hole in the site $i-1$ : note that this is precisely what usually the meanfield approximation does in monomeric lattice gases, where correlations are broken factorizing the density: $\left\langle n_{i} n_{i+1}\right\rangle=\left\langle n_{i}\right\rangle$ $\times\left\langle n_{i+i}\right\rangle$. In Appendix $\mathrm{A}$ we show that this assumption yields a closed expression for the joint probability:

$$
p(i, 0 ; i-1,0)=\frac{p(i-1,0) p(i, 0)}{1-p(i, 2)} .
$$

Eliminating the probabilities for single empty sites and collecting results, the rate equation for the lead head density reads 


$$
\partial_{t} \rho_{i}=\omega_{A} \frac{\left(1-\rho_{i-1}-\rho_{i}\right)\left(1-\rho_{i}-\rho_{i+1}\right)}{1-\rho_{i}}-\omega_{D} \rho_{i} .
$$

As a next step we perform a continuum limit. Relabeling position as a fraction of the system size, $x \equiv i / N$, the density $\rho_{i}$ becomes a function $\rho(x)$ in the mesoscopic limit $N \rightarrow \infty$. The average density at the neighboring sites are obtained by expansion in powers of the lattice constant $a=1 / N$ :

$$
\rho(x \pm a)=\rho(x) \pm a \partial_{x} \rho(x)+\frac{1}{2} a^{2} \partial_{x}^{2} \rho(x)+O\left(a^{3}\right) .
$$

Introducing a new rescaled time $\tau=a t$ and eliminating the single site on and off rates $\omega_{A}$ and $\omega_{D}$ in favor of the gross kinetic rates $\Omega_{A}$ and $\Omega_{D}$ we obtain the rate equation in the mesoscopic limit:

$$
\partial_{\tau} \rho=\Omega_{A} \frac{(1-2 \rho)^{2}}{1-\rho}-\Omega_{D} \rho+O\left(a^{2}\right) .
$$

It is natural to expect a density limited to the range $[0,1 / 2]$, because there cannot be more than one lead head per site. In the stationary regime the physical solution corresponds to the constant density $\rho_{I}$, Eq. (1), determined solely by the binding constant $K=\Omega_{A} / \Omega_{D}$. This value of the isotherm is consistent with the one obtained by McGhee and Von Hippel [26] for the general case of $\ell$-mers.

\section{Tonks gas approach}

To corroborate the results presented above, we compare the isotherm of the rate equation with the one computed from the grand canonical partition function of the Tonks gas. In particular, we shall identify the fugacity $z$ with the binding constant $K$.

The statistical mechanics of the Tonks gas on an open lattice consists of distributing extended objects on the $N$ sites, respecting the excluded volume constraint. In the case of the canonical ensemble this reduces to the combinatorial problem of counting the number of ways to distribute $n$ dimers on $N$ lattice sites. Using the standard trick to represent the two occupied sites of each dimer by a "stick" and the $N-2 n$ empty sites by "balls" [25], one immediately concludes

$$
Z(n, N)=\left(\begin{array}{c}
N-n \\
n
\end{array}\right)
$$

The corresponding grand canonical partition function is readily evaluated using the previous result,

$$
\mathcal{Z}_{N}=\sum_{n=0}^{N} z^{n} Z(n, N)=\frac{(\sqrt{1+4 z}+1)^{N+1}+(\sqrt{1+4 z}-1)^{N+1}}{2^{N+1} \sqrt{1+4 z}},
$$

where $z$ denotes the fugacity [40]. The average density is then obtained in the thermodynamic limit by

$$
\rho=\lim _{N \rightarrow \infty} \frac{1}{N} z \frac{\partial}{\partial z} \ln \mathcal{Z}_{N}=\frac{1}{2}\left(1-\frac{1}{\sqrt{4 z+1}}\right) .
$$

Since the master equation of pure on and off kinetics fulfills detailed balance, the stationary state is given in terms of the grand canonical ensemble. By a single detachment event a configuration $\mathcal{C}$ of $n$ dimers connects to some new configurations $\mathcal{C}^{\prime}$ of $n-1$ dimers. For such configurations the detailed balance condition implies $P(\mathcal{C}) / P\left(\mathcal{C}^{\prime}\right)=\omega_{A} / \omega_{D}=K$. Conversely, the ratio can be determined also from the grand canonical Boltzmann factors. Since, apart from the total exclusion, energy does not enter the problem, the probabilities are determined by the number of dimers only: $P(\mathcal{C})=z^{n} / \mathcal{Z}_{N}$ and $P\left(\mathcal{C}^{\prime}\right)=z^{n-1} / \mathcal{Z}_{N}$. Combining both expressions, we conclude $z=K$ as in the case of monomers.

The last result shows that the "isotherm" obtained using ad hoc approximations (Sec. IV A 1) is at least consistent with thermodynamics. Let us mention that in equilibrium the situation is probably better than in the general case, since correlations are quickly washed out by the coupling to the reservoir. The relaxation towards equilibrium can be rather different from the naive picture of rate equations, see, e.g., Ref. [42].

\section{B. TASEP of dimers}

In pure TASEP particles cannot leave or enter the track except at the boundaries. Correspondingly in the bulk a conservation law holds,

$$
\partial_{t} \rho_{i}=j_{i-1}-j_{i}
$$

The currents $j_{i}$ can be determined by probabilistic arguments. A hopping event of a dimer at site $i$ requires first that site $i$ is occupied by a lead head and second that site $i+1$ is empty. Since we fixed the hopping rate to unity this yields

$$
j_{i}=p(i, 2 ; i+1,0) \text {. }
$$

Hence also in the case of pure TASEP the master equation induces a whole hierarchy of joint probabilities. To close the equation we rely on the same ad hoc approximation for the conditional probabilities as for the on and off kinetics. In Appendix A we show that this implies for the current a closed expression,

$$
j_{i}=\frac{\rho_{i}\left(1-\rho_{i+1}-\rho_{i+2}\right)}{1-\rho_{i+1}} .
$$

Performing the continuum limit as in Eq. (9) one obtains

$$
\partial_{\tau} \rho=-\partial_{x}\left[\frac{\rho(1-2 \rho)}{1-\rho}+\frac{1-2 \rho^{2}}{(1-\rho)^{2}} \frac{a}{2} \partial_{x} \rho+O\left(a^{2}\right)\right] .
$$

Since, at the very end, we consider large but finite systems we have kept the leading correction in $a$, which will turn out to be relevant for the formation of shocks and boundary layers.

The equation of continuity in the bulk has to be supplemented with appropriate boundary conditions. Relying again on the same ad hoc assumption for the conditional probability, and performing the continuum limit, one finds

$$
\rho(0)=\frac{\alpha}{1+\alpha},
$$



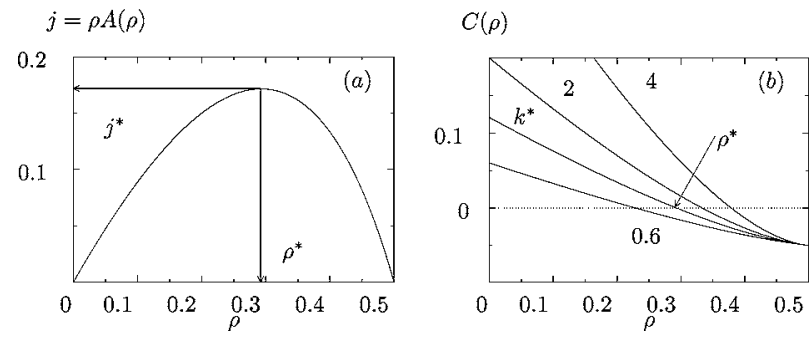

FIG. 3. (a) Graph of the current-density relation $\rho A(\rho)$ in Eq. (20). (b) Graph of the source-drain $C(\rho)$ term in the Smoluchowski equation (20) for different values of $K$ and $\Omega_{D}=0.1$.

$$
\rho(1)=\frac{1-\beta}{2} \text {. }
$$

The details are presented in Appendix B. The properties of these equations have been studied in detail in Ref. [16]. The resulting phase diagram is topologically equivalent to the one of TASEP of monomers. The continuum analog for the current-density relation, Eq. (15), reads

$$
j=\frac{\rho(1-2 \rho)}{1-\rho}
$$

and exhibits a maximum at a distinguished density $\rho^{*}$ $=1 /(2+\sqrt{2})$. This value will play an important role in the more general case of TASEP coupled to on and off kinetics.

\section{Combining TASEP and on and off kinetics}

As anticipated at the beginning of this section, we identify the current and the source terms of Eq. (4) and we reexpressed everything in terms of the density. The current is described by Eq. (18) and the on and off kinetics by Eq. (9). By taking the mesoscopic limit we have introduced the gross attachment and detachment rates and rescaled the time consistently in both processes. The balance equation to order $a$ can be easily rewritten as

$$
\partial_{\tau} \rho=-\partial_{x}\left[-\frac{a}{2} \frac{\partial_{x} \rho}{(1-\rho)^{2}}+\frac{\rho(1-2 \rho)}{1-\rho}\right]+\Omega_{A} \frac{(1-2 \rho)^{2}}{(1-\rho)}-\Omega_{D} \rho
$$

with the boundary conditions, Eq. (17). These equations constitute the mean-field description to be discussed in the following section.

Equation (19) may also be recast in a form reminiscent of a nonlinear Smoluchowski equation [43]:

$$
\partial_{\tau} \rho=-\partial_{x}\left[A(\rho) \rho-B(\rho) \rho \partial_{x} \rho\right]+C(\rho),
$$

where the coefficients $A, B$, and $C$ depend on $\rho$. The second coefficient will prove not to be relevant since it scales to zero with increasing the system size to infinity.

We may now interpret $j(\rho)=\rho A(\rho)$ as an effective particle current and $C(\rho)$ as a source or drain term; see Fig. 3 for an illustration. As will become clear in the following section the maximum of $j(\rho)$ and the zeros of $C(\rho)$ play an important role for the analytical form of the stationary solution.

\section{SOLUTION OF THE MEAN-FIELD EQUATION AND PHASE DIAGRAM}

This section is devoted to a discussion of the phase diagram emerging from combining TASEP of dimers with on and off kinetics. We shall derive density profiles within a mean-field approach, identify different phases and phase boundaries as the kinetic rates are varied. In particular, we demonstrate the role of the binding constant in the topological changes of the phase diagram as a certain critical value is reached.

\section{A. Emergence of shocks and boundary layers}

The mean-field equation for the stationary density profile, Eq. (19), supplemented with $\partial_{\tau} \rho \equiv 0$, is a second order differential equation as long as the lattice constant $a$ is small but finite. In the limit $a \searrow 0$ the differential equation degenerates into a first order one. However, the density profile has to match the two boundaries, and it appears that the problem is overdetermined. Indeed one can construct separately a density profile fulfilling either the left or right boundary condition. The two branches will be referred to as $\rho_{\alpha}$ and $\rho_{\beta}$, respectively. In general, the two branches do not join smoothly together. One expects the solution of the second order differential equation to be well approximated by the two branches $\rho_{\alpha}$ and $\rho_{\beta}$ except for a region where the solution rapidly crosses over from one branch to the other. Upon decreasing the lattice constant $a$ the crossover region shrinks leading eventually to a discontinuity of the density profile for $a=0$. Depending on the position of such a discontinuity $x_{w}$ we refer to it as a shock $\left(0<x_{w}<1\right)$ or a boundary layer $\left(x_{w}=0\right.$ or $\left.x_{w}=1\right)$.

To locate the position of the shock or boundary layer we rely on a very general argument already used in Refs. $[22,41]$. The second order differential equation for the stationary density profile can be written in the form of a balance equation $\partial_{x} j=F_{A}-F_{D}$, where the current along the track reads

$$
j=-\frac{a}{2} \frac{\partial_{x} \rho}{(1-\rho)^{2}}+\frac{\rho(1-2 \rho)}{1-\rho} .
$$

Integrating over a small region of width $2 \delta x$ around the shock one obtains

$$
j\left(x_{w}+\delta x\right)-j\left(x_{w}-\delta x\right)=\int_{x_{w}-\delta x}^{x_{w}+\delta x}\left(F_{A}-F_{D}\right) d x .
$$

In the limit $a \searrow 0$ the left-hand side reduces to $j_{\alpha}\left(x_{w}+\delta x\right)$ $-j_{\beta}\left(x_{w}-\delta x\right)$ [where we have defined $j_{\alpha}=\rho_{\alpha}\left(1-2 \rho_{\alpha}\right) /(1$ $\left.-\rho_{\alpha}\right)$ as the current set by the left boundary and similarly for the right one $j_{\beta}$ ]. Performing the limit $\delta x \searrow 0$ the right-hand side of Eq. (22) vanishes and yields the matching rule in terms of the currents,

$$
j_{\alpha}\left(x_{w}\right)=j_{\beta}\left(x_{w}\right) .
$$

Equivalently the rule implies for the densities at the matching point, 


$$
\rho_{\alpha}\left(x_{w}\right)=\frac{1}{2} \frac{1-2 \rho_{\beta}\left(x_{w}\right)}{1-\rho_{\beta}\left(x_{w}\right)} .
$$

Let us make a comment. The fact that the in-track current is continuous at the shock is consistent with the idea of the mesoscopic limit. The fluxes due to attachment and detachment are important only on the length scale of the system size. Locally, i.e., on the scale of the lattice constant, the balance equation is dominated entirely by the unidirectional hopping process (TASEP).

\section{B. Analytical solution}

The left and right branches of the stationary density profile are determined by solving Eq. (19), once we set the lefthand side to zero and discard the second order derivative, i.e.,

$$
\frac{1}{\Omega_{D}} \partial_{x}\left[\frac{\rho(1-2 \rho)}{1-\rho}\right]=K \frac{(1-2 \rho)^{2}}{(1-\rho)}-\rho
$$

(where the binding constant $K=\Omega_{A} / \Omega_{D}$ has been introduced), which has to be supplemented by the appropriate boundary conditions, Eq. (17). By separation of variables the general solution $G(\rho)=x+$ const is obtained after a straightforward integration with

$$
G(\rho)=\frac{1}{\Omega_{D} K}\left[\ln (1-\rho)+A_{+} \ln \left(\rho_{+}-\rho\right)+A_{-} \ln \left|\rho_{-}-\rho\right|\right],
$$

where we introduced the zeros of the source term

$$
\rho_{ \pm} \equiv \frac{1}{2}\left(1 \pm \frac{1}{\sqrt{1+4 K}}\right)
$$

and the amplitudes

$$
A_{ \pm} \equiv \frac{K}{1+4 K} \frac{1-4 \rho_{ \pm}+2 \rho_{ \pm}^{2}}{\left(1-\rho_{ \pm}\right)\left(\rho_{ \pm}-\rho_{\overline{+}}\right)} .
$$

The function $G(\rho)$ exhibits a singularity in the physical regime $0 \leq \rho \leq 1 / 2$ at the isotherm of the on and off kinetics $\rho_{I}=\rho_{-}$. One easily checks that $G(\rho)$ exhibits an extremum when the corresponding current, Eq. (18), is maximal, i.e., at $\rho=\rho^{*}$. For $\rho_{I}>\rho^{*}\left(\rho_{I}<\rho^{*}\right)$ the extremum of $G(\rho)$ corresponds to a maximum (minimum). A change of topology of the graph of the solution occurs once both distinguished densities coincide $\rho_{I}=\rho^{*}$, which happens at a critical value of the binding constant $K^{*}=(1+\sqrt{2}) / 2$. At this special value the amplitude $A_{-}$vanishes and the function $G(\rho)$ becomes smooth and monotonic in the physical regime $0<\rho<1 / 2$.

After matching the boundary conditions, the left and right solutions are obtained up to inversion of a function

$$
\begin{gathered}
L_{\alpha}\left(\rho_{\alpha}\right) \equiv G(\rho)-G[\alpha /(1+\alpha)]=x, \\
R_{\beta}\left(\rho_{\beta}\right) \equiv G(\rho)-G[(1-\beta) / 2]+1=x .
\end{gathered}
$$

Upon inverting, the singularity of $G(\rho)$ transforms into a horizontal asymptote, whereas the extremum manifests itself
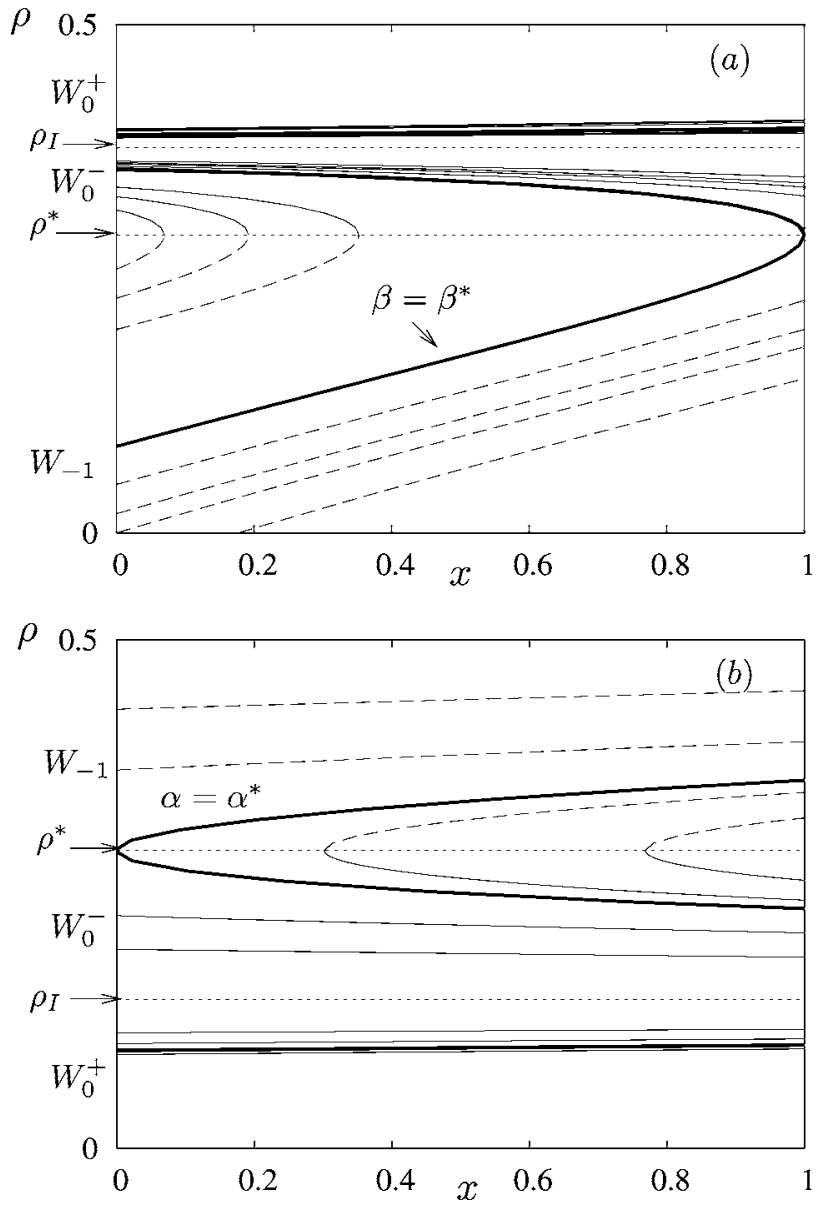

FIG. 4. The functions $W_{-1}$ (dashed lines), $W_{0}$ (full lines), $W_{0}^{+}$ (full lines) for (a) $K=4, \Omega_{D}=0.05$ (high binding affinity) and (b) $K=0.25, \Omega_{D}=0.05$ (low binding affinity). The branches $W_{0}^{+}$and $W_{0}^{-}$ are separated by the isotherm density $\rho_{I}$. Varying $\alpha$ or $\beta$ results in a simple shift parallel to the $x$ axis. In (a) and (b) the thick lines represent the solutions where the branch point hits the right and left end of the system. The dotted lines indicate the critical density value $\rho^{*}$ (where branch point occurs) and the isotherm $\rho_{I}$.

as a branch point, see Fig. 4. The functions are multivalued with three branches, to be referred to as $W_{0}^{+}, W_{0}^{-}$, and $W_{-1}$.

\section{High binding affinity: $K>K^{*}$}

For large binding constants, $K>K^{*}$, the equilibrium density of the on-off isotherm is larger than the critical density at which the current of pure TASEP is maximal, $\rho_{I}>\rho^{*}$, see Fig. 4.

For densities smaller than $\rho_{I}$, the track accumulates particles from the reservoir, since

$$
F_{A}-F_{D}=\Omega_{D} \frac{1+4 K}{1-\rho}\left[\left(\rho-\rho_{-}\right)\left(\rho-\rho_{+}\right)\right] .
$$

Hence the isotherm acts as an attractor. This property has to be reflected in the left and right solutions of the balance equation for the current, $\partial_{x} j=\left(\partial_{\rho} j\right)\left(\partial_{x} \rho\right)=F_{A}-F_{D}$. For $\rho(0)$ $=\alpha /(1+\alpha)>\rho^{*}$, the branches $W_{0}^{ \pm}$are unstable. For densities $\rho>\rho_{I}$ the coupling to the reservoir induces a decrease in 


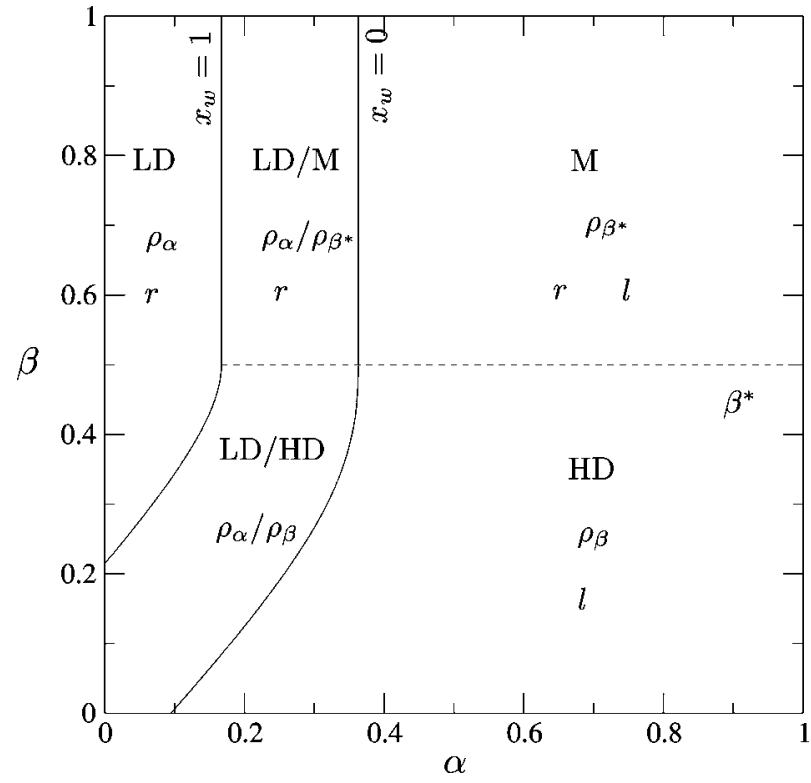

FIG. 5. High binding affinity; phase diagram for $K=2, \Omega_{D}$ $=0.1$. In the low density (LD) phase the profile is given by the left solution $\rho_{\alpha}$ and a boundary layer appears at the right $(r)$. The position of boundary layers $(r, l)$, as well as the relevant bulk solution $\left(\rho_{\alpha}, \rho_{\beta}\right.$, and $\left.\rho_{\beta^{*}}\right)$ are indicated for all phases (HD: high density phase; LD/HD: low density-high density coexistence phase; $M$ : Meissner phase; LD/M: low density-Meissner coexistence phase, see text).

density, whereas $W_{0}^{+}$is increasing. Similarly for $\rho^{*}<\rho<\rho_{I}$ the net gain due to the on and off kinetics is not reflected by the decreasing function $W_{0}^{-}$. One concludes that for the left solution the only physical branch is $W_{-1}$. For $\alpha>\alpha^{*}$ $=\rho^{*} /\left(1-\rho^{*}\right)=1 /(1+\sqrt{2})$, i.e., the critical entrance rate of dimer TASEP, a left boundary layer necessarily appears.

For the right solution the argument is just reversed, i.e., the physical branches are $W_{0}^{ \pm}$. The critical exit rate $\beta^{*}$ of dimer TASEP is determined by $\rho(1)=\left(1-\beta^{*}\right) / 2=\rho^{*}$ and equals $\beta^{*}=1 /(1+\sqrt{2})$. For exit rates larger than the critical one a right boundary layer emerges. The complete phase diagram is obtained by identifying different scenarios to match left and right solutions. The boundary conditions, Eq. (17), can be satisfied by the stable branches of the left and right solution only for $\alpha<\alpha^{*}, \beta<\beta^{*}$, respectively. The merging of the two solutions by Eq. (24) is achieved at some position $x_{w}$. Depending on whether the (i) $0<x_{w}<1$, (ii) $x_{w}<0$, and (iii) $x_{w}>1$, a domain wall in the bulk emerges (i), a boundary layer at the left (ii) or right (iii) appears. For $\beta>\beta^{*}$ an additional boundary enters the system. The result of such an analysis is presented in Fig. 5, and the chain of arguments follows the one of Ref.[41].

For example, for large entrance rate $\alpha$ and low exit rates $\beta$ the density profile is in a high density phase (HD), i.e., the solution $\rho(x)$ exceeds $\rho^{*}$, and is given by the stable right solution $\rho_{\beta}(x)$. At the left end $x=0$ a boundary layer appears. Similarly a region of a low density phase (LD) and low density-high density phase coexistence (LD/HD) with appropriate boundary layer and domain wall is inferred from the figure.
For $\beta>\beta^{*}$ the right solution is replaced by the critical right solution $\rho_{\beta=\beta^{*}}$, which constitutes the analog to the maximal current phase in TASEP: the system indeed cannot transport more than the current $j_{\beta^{*}}$ imposed by the critical exit rate $\beta^{*}$.

Changing the exit rate beyond $\beta^{*}$ does not affect the density profile except in a small boundary layer. This phenomenon is similar to a superconductor where an external magnetic field does not enter the sample except for a short penetration depth. This analogy suggests to denote this region in the phase diagram by $M$ (Meissner) phase. The coexistence of low-density and Meissner phase (LD/M) extends the coexistence phase (LD/HD) for $\beta>\beta^{*}$.

The phase boundaries can be computed analytically up to inversion of a function. For example, the HD-LD/HD phase boundary is obtained by requiring the domain wall to fall on the left end of the system $x_{w}=0$. Thus the continuity condition, Eq. (24), translates the boundary condition $\rho(0)=\alpha /(1$ $+\alpha)$ to $\rho(x+0)=(1-\alpha) / 2$. In terms of the implicit solution, Eq. (28b), this implies

$$
R_{\beta}\left(\frac{1-\alpha}{2}\right)=0 .
$$

Similarly the LD-LD/HD phase boundary is determined by placing the domain wall at $x_{w}=1$, which yields

$$
L_{\alpha}\left(\frac{\beta}{1+\beta}\right)=1 .
$$

For $\beta>\beta^{*}$ the preceding equations still hold provided $\beta$ is replaced by the critical exit rate $\beta^{*}$ [45]. Correspondingly, phase boundaries degenerate to straight vertical lines.

Density profiles exemplifying different regions of the phase diagram are shown in Fig. 6 and compare nicely to the corresponding simulated data.

The low density phase disappears from the phase diagram upon increasing $\Omega_{D}$ at fixed $K$. Such a topological change occurs, once the phase boundary of the LD/ $M$ coexistence phase hits the $\beta$ axis of the phase diagram (i.e., $\alpha=0$ ). The condition is inferred from Eq. (31) and reads

$$
L_{\alpha=0}\left(\frac{\beta^{*}}{1+\beta^{*}}\right)=1 .
$$

A numerical solution of the preceding equation for $\Omega_{D}^{*}$ as a function of $K$ is presented in Fig. 7.

In the limit $\Omega_{D} \rightarrow 0^{+}$the $\mathrm{LD} / \mathrm{HD}$ coexistence phase shrinks continuously to the line $\alpha=\beta$ and one recovers the dimer TASEP phase diagram. For $\Omega_{D} \gg \Omega_{D}^{*}$, the coexistence phases constitute only a marginal region in the $\alpha$ - $\beta$ plane, located close to the $\beta$ axis. The phase diagram is dominated by the HD and $M$ phases, however, the density profile approaches the constant value $\rho_{I}$ in the bulk, as expected from pure on-off kinetics [46].

\section{Low binding affinity: $K<K^{*}$}

If $K<K^{*}$ the functional form of the solutions is still given by Eqs. (26), (28a), (28b). However, now the isotherm $\rho$ $=\rho_{I}$ lies below the critical density, $\rho_{I}<\rho^{*}$. By the same rea- 

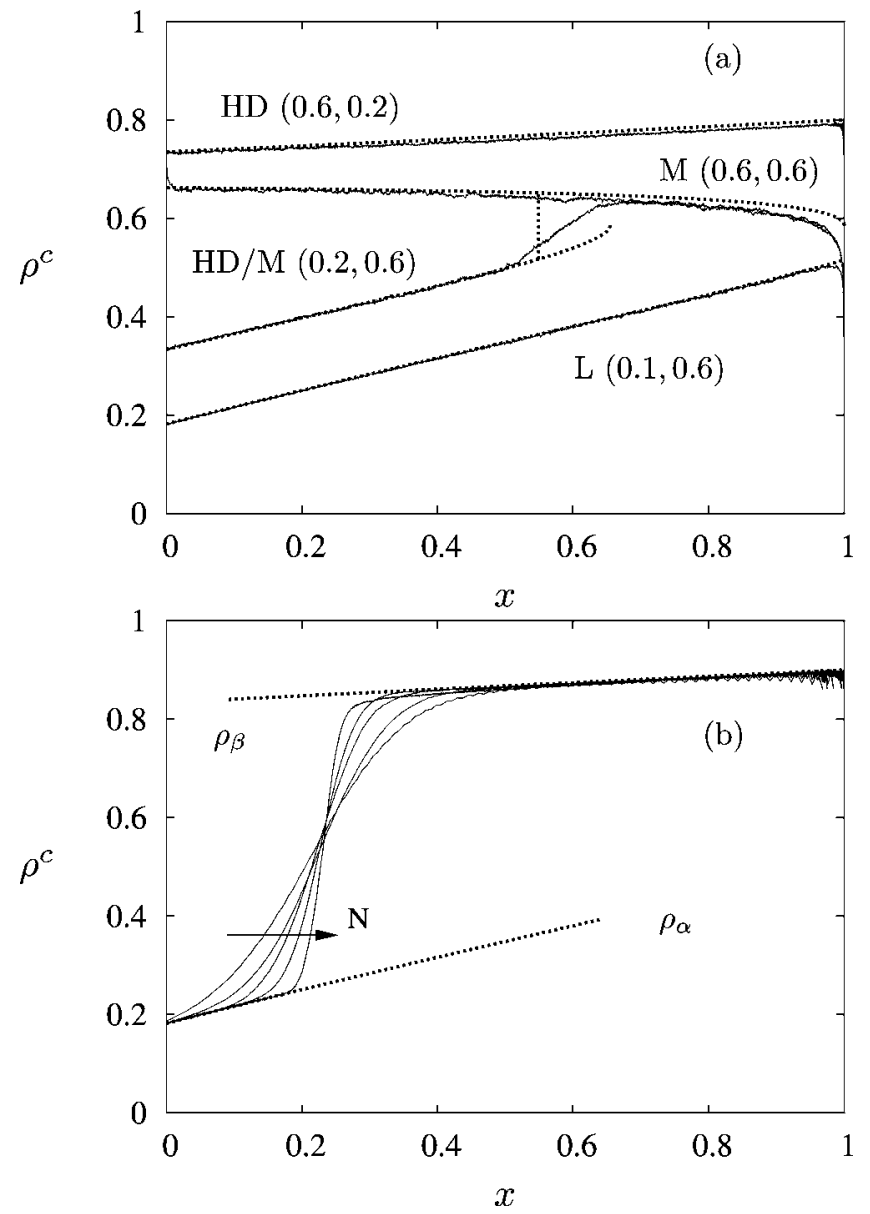

FIG. 6. (a) Density profiles for $K=2$ and $\Omega_{D}=0.1$ in systems of $N=4096$ lattice sites; parameters $(\alpha, \beta)=(0.1,0.6), \quad(0.2,0.6)$, $(0.6,0.6),(0.6,0.2)$. (b) Approach of the mesoscopic limit of the density profile for $\alpha=\beta=0.1, \Omega_{D}=0.1, K=2$ and different system sizes $(N=128,256,512,1024,4096)$; the domain wall becomes steeper with the increasing of $N$. Wiggly lines represent simulated data, while dotted lines indicate analytic solutions.

soning as above, see Eq. (29), the left stable branches are the ones which approach the isotherm, $W_{0}^{ \pm}$in Fig. 4(b), while the only stable solution for the right side is $W_{-1}$, i.e., reversed to $K>K^{*}$.

Using the same methods as for the high binding constant, phases and phase boundaries are identified by matching the appropriate left and right solutions. A cut of the parameter space at fixed $\Omega_{D}$ and $K$ is presented in Fig. 8. The high density phase disappears from the phase diagram for detachment rates exceeding a critical value $\Omega_{D}^{*}(K)$, see Fig. 7 [47].

Let us stress that there is an important difference with respect to the monomeric analog, namely the particle-hole symmetry is no longer present. Correspondingly, a symmetry transformation does not yield the phase diagram of the "low affinity" from the one of the "high affinity." Yet, the exchange of high densities and low densities, left and right, exit by entrance rates yields the correct topology of the phase diagram as well, as qualitatively the density profile.

\section{E. Critical case $K=K^{*}$}

The limiting case when the binding constant equals the critical one, $K=K^{*}$, requires a separate analysis, since the

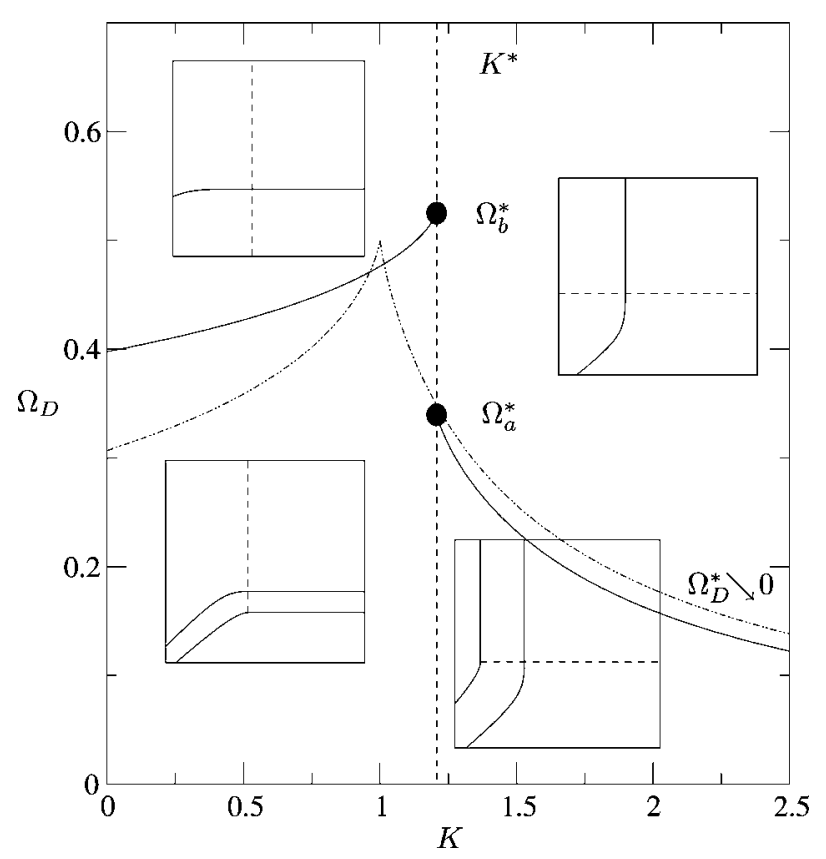

FIG. 7. Critical detachment rate $\Omega_{D}^{*}$ as a function of the binding constant $K$ [solid line shows $\Omega_{D}^{*}(K)$ for dimers, dotted line for monomers]. The dashed line $K^{*}$ separates the cases of "high" from the "low" binding affinity (for dimers). For large $K$ the critical detachment rate approaches zero. Insets represent the different topologies of the $\alpha$ - $\beta$ section of the phase diagram.

amplitude $A_{-}$vanishes, canceling exactly the logarithmic divergence at $\rho=\rho_{-}\left(\right.$nota bene $\left.\rho_{-}=\rho^{*}=\rho_{I}\right)$. This signals that $\rho=\rho^{*}=$ const is a spurious solution of the balance equation for the stationary density profile. It corresponds to the maximal current (MC) phase of pure dimer TASEP. Indeed for $K=K^{*}$ Eq. (25) simplifies to

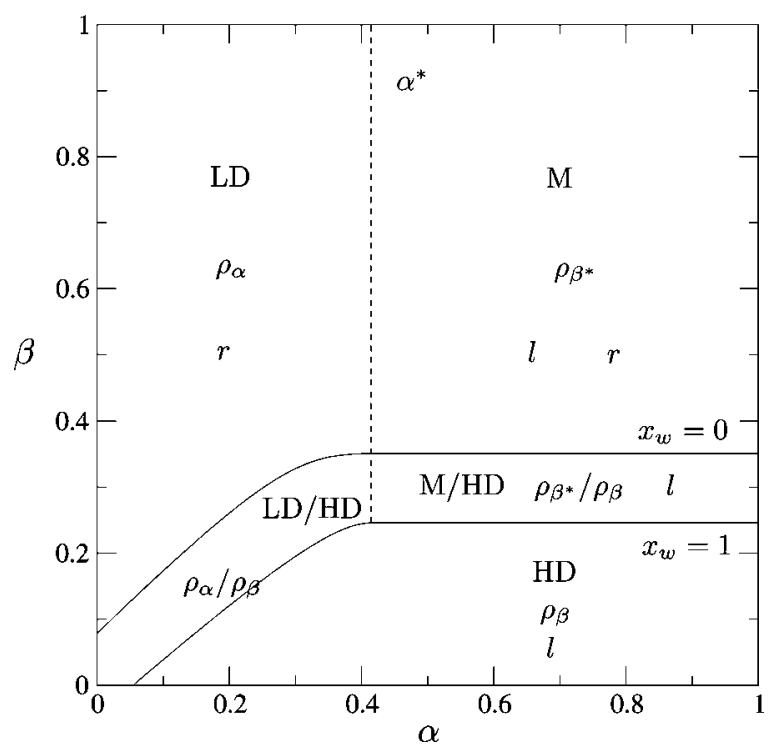

FIG. 8. Low binding affinity; phase diagram for $K=0.8, \Omega_{D}$ $=0.1$. Legend as in Fig. 5 . 


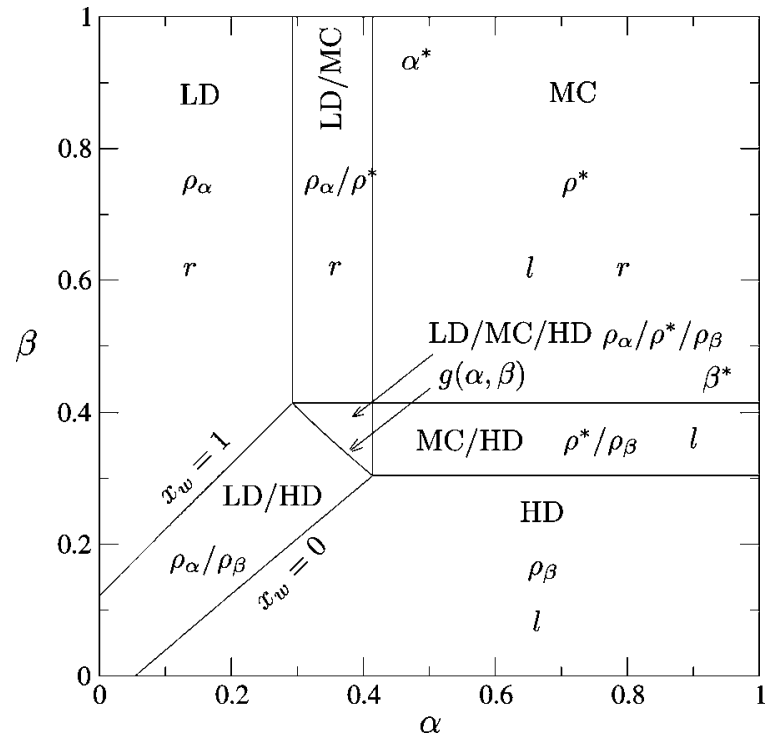

FIG. 9. Critical case; phase diagram for the case $K=K^{*}, \Omega_{D}$ $=0.1$. Seven phases appear: LD, MC, HD, and all the possible coexistence: LD/MC, MC/HD, LD/HD, LD/MC/HD. Legend as in Fig. 5.

$$
\left(\rho-\rho^{*}\right)\left[\frac{2 \rho-(2+\sqrt{2})}{(1-\rho)} \partial_{x} \rho-\Omega_{D}\left(\rho-\frac{1}{\sqrt{2}}\right)\right]=0
$$

The nontrivial solution Eq. (26) specializes to

$$
G(\rho)=\frac{2 \sqrt{2}-2}{\Omega_{D}}\left[\ln (1-\rho)-\sqrt{2} \ln \left(\frac{1}{\sqrt{2}}-\rho\right)\right],
$$

which is a monotonic function in the physical regime. Correspondingly, the inverse function is single valued.

The properties of the phase diagram for $K=K^{*}$ are obtained by assembling the resulting left and right branches, as well as the constant critical density $\rho^{*}$, for different boundary conditions. We have determined numerically the line where the domain wall hits the left and right end, $x_{w}=0$ and $x_{w}=1$, respectively, by solving Eqs. (30) and (31). Moreover, both left and right solutions now can approach the critical one (i.e., $\rho=\rho_{I}$ ) in the bulk and therefore a triple phase coexistence LD-MC-HD is possible. This phase is bounded by the lines $\beta=\beta^{*}$ above and $\alpha=\alpha^{*}$ on the right. Furthermore, the curve $g(\alpha, \beta)$, where the left and right currents match additionally the critical one separates the triple phase coexistence from the LD-HD phase. Implicitly $g(\alpha, \beta)$ is given by the condition $L_{\alpha}\left[\beta^{*} /\left(1+\beta^{*}\right)\right]=R_{\beta}\left[\left(1-\alpha^{*}\right) / 2\right]$.

Depending on the value of the kinetic rate $\Omega_{D}$, different topologies for the phase diagram arise. For example, for low $\Omega_{D}$ seven phases are present in the $\alpha$ - $\beta$ diagram (see Fig. 9). Upon increasing $\Omega_{D}$, a first topological change, analogous to the case of high binding affinity, occurs, i.e., the LD phase exits the phase diagram. By solving Eq. (32), one obtains the critical value $\Omega_{a}^{*} \approx 0.33945$, see Fig. 7. At another critical value $\Omega_{b}^{*} \approx 0.52496$ the HD phase disappears, and at still higher detachment rates $\Omega_{D}>\Omega_{c}^{*}=0.86441$ the LD-HD coexistence phase is no more present and the phase diagram
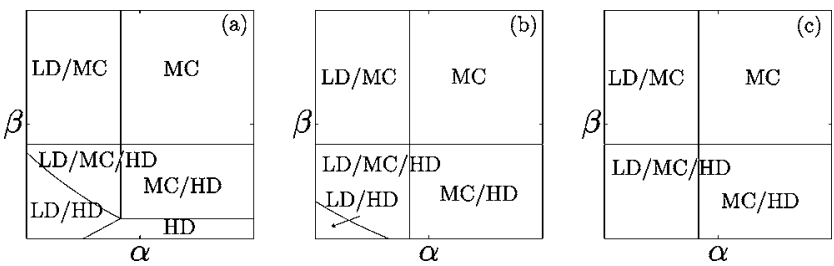

FIG. 10. Different topologies of the phase diagram for $K=K^{*}$ and (a) $\Omega_{a}^{*}<\Omega_{D}<\Omega_{b}$, (b) $\Omega_{b}^{*}<\Omega_{D}<\Omega_{c}^{*}$, and (c) $\Omega_{D}>\Omega_{c}^{*}$. Phases are indicated in the graphs.

(but not the density profiles) is independent of $\Omega_{D}$. Representative graphs of these topologies are shown in Figs. 7 and 10 .

\section{CONCLUSIONS}

We have studied the nonequilibrium dynamics of a 1D driven lattice gas with open boundaries, where individual particles are considered as dimers occupying two lattice sites simultaneously. Upon combining a generalized mean-field theory with extended stochastic simulations, we have explored the phase behavior of the nonequilibrium steady state as a function of the kinetic rates. In particular, we have analyzed the interplay between the coupling of the system to a reservoir at the boundaries and at the bulk.

In the following we compare our results with those obtained for the analogous problem of simple TASEP of monomers coupled to Langmuir kinetics. The balance equation in the stationary state that constitutes the equivalent of Eq. (19) has been derived in Refs. [22,41]:

$$
\frac{1}{\Omega_{D}} \partial_{x}[\rho(1-\rho)]=K(1-\rho)-\rho .
$$

Although the structure of the equation is the same, $\partial_{x} j=F_{A}$ $-F_{D}$, the current and the source terms obey a rather different functional dependence. In particular, the equation exhibits a particle-hole symmetry, i.e., density profiles and the complete phase diagram is symmetric with respect to the following transformation rule $\rho(x) \leftrightarrow 1-\rho(1-x), \Omega_{D} \leftrightarrow \Omega_{A}, \alpha \leftrightarrow \beta$. Also, a TASEP for dimers disregarding the on-off kinetics can be cast in a particle-hole symmetric form, introducing an effective hole density $\bar{\rho}$ by the requirement $j(\rho)=j(\bar{\rho})$ and interchanging $\alpha$ with $\beta$; this explains why the phase diagram is symmetric along the diagonal. Similarly, a pure on-off kinetics is invariant with respect to the transform $\Omega_{A} \leftrightarrow \Omega_{D}$ and an effective hole density $\bar{\rho}=(1-2 \rho)^{2} /(1-\rho)$. However, one may easily show that the two symmetries of simple dimer TASEP and on-off kinetics never coincide for any $K$ $>0$, implying that the coupling of both processes breaks the particle-hole symmetry.

It is remarkable that the topology of the resulting phase diagram is almost unaffected by this broken symmetry. In fact, the single qualitative change of topology occurs at $K$ $=K^{*}$ for intermediate $\Omega_{D}$, see Figs. 7 and $10(\mathrm{~b})$. The origin of the robustness of the picture can be traced back to the following observations: (i) in a mean-field approach the balance equation for the stationary density profile is an autonomous 
TABLE I. Differences and analogies between the model with monomers and dimers: the current-density relation presents in both cases a maximum at $\rho^{*}$ which appears at different values, but the particle-hole symmetry which is trivial in the monomers is actually effective in the dimers; the boundary conditions, as well as the current, need to be set differently in the dimers in order to keep track of their geometry; as a consequence of this the critical values of the boundary rates are different from the critical density in the case of dimers; the isotherm is related to the binding constant in a nontrivial way in the case of dimers; the particle-hole symmetry is preserved by the sources only in the monomers case. See the text for more details.

\begin{tabular}{lcc}
\hline \hline Quantity & Monomers & Dimers \\
\hline Particle dens. & $\rho$ & $\rho$ \\
Coverage dens. & $\rho$ & $2 \rho$ \\
$j$ & $\rho(1-\rho)$ & $\frac{\rho(1-2 \rho)}{1-\rho}$ \\
$F_{A}$ & $\Omega_{A}(1-\rho)$ & $\Omega_{A} \frac{(1-2 \rho)^{2}}{1-\rho}$ \\
$F_{D}$ & $\Omega_{D} \rho$ & $\Omega_{D} \rho$ \\
$\rho(0)$ & $\alpha$ & $\frac{\alpha}{1+\alpha}$ \\
$\rho^{*}(1)$ & $1-\beta$ & $\frac{1-\beta}{2}$ \\
$\rho^{*}$ & $\frac{1}{2}$ & $\frac{1}{\sqrt{2}(1+\sqrt{2})}$ \\
$\alpha^{*}=\beta^{*}$ & $\frac{1}{2}$ & $\frac{1}{1+\sqrt{2}}$ \\
$j^{*}$ & $\frac{1}{4}$ & $\frac{1}{(1+\sqrt{2})^{2}}$ \\
$j_{\alpha}$ & $\alpha(1-\alpha)$ & $\frac{\alpha(1-\alpha)}{1+\alpha}$ \\
$j_{\beta}$ & $\beta(1-\beta)$ & $\frac{\beta(1-\beta)}{1+\beta}$ \\
$\rho_{I}$ & $\frac{K}{1+K}$ & $\frac{1}{2}\left(1-\frac{1}{\sqrt{1+4 K}}\right)$ \\
$K^{*}$ & 1 & $\frac{1}{2}(1+\sqrt{2})$ \\
\hline \hline
\end{tabular}

first order ordinary differential equation; (ii) the currentdensity relation exhibits a single extremum for both cases [48]; (iii) the solution $\rho(x)$ is obtained by quadrature and inversion of a function resulting in a multivalued function with a simple branch point (due to the maximum in the current-density relation) and a horizontal asymptote (due to the on-off isotherm). Although the shape of the explicit solution depends on the details of the density current relation and the functional form of the on-off kinetics, the overall topological features remain unchanged. This reasoning explains that particle hole symmetry is relevant for the topology of the phase diagram only if the branching point is canceled by a zero of the on-off kinetics, i.e., precisely at $K$ $=K^{*}$. We conclude that the scenario for $K=K^{*}$ presented in Fig. 10 generalizes the monomer TASEP coupled to Langmuir kinetics. Nevertheless, there are many differences in the numerical values of several quantities, which are summarized in Table I.

An interesting difference between monomers and dimers resulting from the broken particle-hole symmetry can be read off from Fig. 7, where the change of the phase diagram topology is shown as a function of $\Omega_{D}$ and $K$. For monomers not only the critical value $K^{*}$ is shifted, but the line $\Omega_{D}(K)$ is continuous. Both the lines $\Omega_{D}=\Omega_{D}^{*}$ and $K=K^{*}$ mark continuous transitions, but they concern two different events: while at $K=K^{*}$ the shape of the analytical solution changes, at
$\Omega_{D}=\Omega_{D}^{*}$ a domain wall enters the system. In the monomers the critical detachment rates $\Omega_{a}^{*}$ and $\Omega_{b}^{*}$ coincide and the topology of the phase diagram in Fig. 7 is such that it is impossible to go from a configuration as in the bottom-left to the one in the top-right by changing $K$ without crossing the transition line $\Omega_{D}^{*}$. The (continuous) transition occurring at $\Omega_{D}=\Omega_{D}^{*}$ can be studied experimentally since the density profiles are remarkably different.

It is possible to generalize the continuous equation to the case of a system containing $\ell$-mers, using directly the continuous results presented in Refs. [16,26]:

$$
\frac{1}{\Omega_{D}} \partial_{x}\left[\frac{\rho(1-\ell \rho)}{1-(\ell-1) \rho}\right]=K \frac{(1-\ell \rho)^{\ell}}{[1-(\ell-1) \rho]^{\ell-1}}-\rho .
$$

The qualitative picture is contained already in the case of dimers; although the functional form of the solution depends on the size of the $\ell$-mers, the scenario of three branches, characterized by the maximum of the current-density relation $\rho^{*}$ and the horizontal asymptote $\rho_{I}$, is robust.

In conclusion, we have studied a driven one-dimensional lattice gas of dimers where the dynamics of the totally asymmetric exclusion process has been coupled to the on-off kinetics in the bulk. We used the ad hoc "refined" mean-field for the TASEP part introduced in previous works $[3,15,16]$ and proved that it is consistent with the assumptions made for the on-off part. The main effect of extended nature of dimers on the phase behavior of the system is related to the breaking of a symmetry in the problem (particle hole). This does have quantitative but not qualitative consequences on the density profile and on the phase diagram. The origin of the robustness of the picture found for monomers can be traced back to the form of the stationary density profile which depends exclusively on the form of the current-density relation and of the isotherm of the on-off kinetics. This suggests that the TASEP dynamics washes out the interesting two-step relaxation dynamics that characterizes the on-off kinetics of dimers. The nontrivial outcome is that, in these systems, the diffusion (yet asymmetric) always dominates the large time-scale relaxation. Further studies on the dynamical correlation functions could point out more subtle differences between the dynamics of dimers and monomers. We conjecture that quite interesting phenomena would arise upon taking into account other interactions between the particles, which suggest to consider alternative current-density relations (with more maxima) or nontrivial on-off isotherm (cooperative on-off kinetics).

\section{ACKNOWLEDGMENTS}

We have benefited from discussions with Jaime Santos, Andrea Parmeggiani, Mauro Mobilia, Hauke Hinsch, and Tobias Reichenbach.

\section{APPENDIX A: CONDITIONAL PROBABILITY}

In this appendix we derive an approximate expression for the joint probability $p(i, 0 ; i-1,0)$ and $p(i, 2 ; i+1,0)$ starting from the mean-field assumption 


$$
p(i, 0 \mid i-1,0) \simeq p[i, 0 \mid(i-1,0) \vee(i-1,2)] .
$$

Since the event $(i, 0 ; i-1,1)$ is excluded by the dimer geometry, the mean-field approximation assumes the occupancy of site $i$ to be independent of the state of the previous site. Using Bayes theorem, the right-hand side of Eq. (A1) can be expressed as

$$
\begin{aligned}
& p[(i, 0) \mid(i-1,2) \vee(i-1,0)] \\
& \quad=\frac{p[(i-1,0) \vee(i-1,2) \mid(i, 0)] p(i, 0)}{p[(i-1,0) \vee(i-1,2)]} .
\end{aligned}
$$

The conditional probability in the numerator is unity, $p[(i$ $-1,0) \vee(i-1,2) \mid(i, 0)]=1$, since a trailhead cannot occupy site $i-1$ if site $i$ is empty. Moreover, the events $(i, 0)$ and $(i, 2)$ in the denominator are mutually exclusive, $p[(i$ $-1,0) \vee(i-1,2)]=p(i-1,0)+p(i-1,2)$. Collecting results and using Eq. (A1)

$$
p(i, 0 \mid i-1,0)=\frac{p(i, 0)}{p(i-1,0)+p(i-1,2)} .
$$

The normalization condition compatible with the geometry of the dimers allows us to rewrite the denominator, $p(i$ $-1,0)+p(i-1,2)=1-p(i-1,1)=1-p(i, 2)$, and one obtains Eq. (6).

A similar argument can be used to compute the joint probability $p(i, 2 ; i+1,0)$, using $p(i+1,0 \mid i, 2)$ under the same initial hypothesis

$$
p(i+1,0 \mid i, 2) \simeq p[i+1,0 \mid(i, 0) \vee(i, 2)] .
$$

Using this equation and the normalization condition $p(i$ $+1,0 \mid i, 0) p(i, 0)+p(i+1,0 \mid i, 1) p(i, 1)+p(i+1,0 \mid i, 2) p(i, 2)$ $=p(i+1,0)[$ together with the fact that $p(i+1,0 \mid i, 1)=0$ for obvious geometric reasons], one can show that $p(i$ $+1,0 \mid i, 2) \simeq p(i+1,0 \mid i, 0)$.

\section{APPENDIX B: BOUNDARY CONDITIONS}

We recall the arguments given previously $[3,15,16]$ to justify the boundary conditions, Eq. (17).

Left boundary. The left boundary conditions in the continuum limit can be derived from the rate equation. The incoming flux is given by the entrance rate multiplied by the probability of having the first two sites free, which is derived from the normalization condition on site $i=2$ (given that site $i=1$ can never contain a trailhead):

$$
p(1,0 ; 2,0)=1-p(2,2)-p(2,1)=1-\rho_{2}-\rho_{3},
$$

while the outgoing flux is determined by the usual relation. This yields to

$$
\partial_{\tau} \rho_{1}=\alpha\left(1-\rho_{2}-\rho_{3}\right)-\frac{\rho_{2}\left(1-\rho_{3}-\rho_{4}\right)}{1-\rho_{3}} .
$$

In the continuum limit (in first approximation) of the stationary state $\rho_{2}=\rho_{3}=\rho$ and therefore the equation can be solved for $\rho$ in terms of $\alpha$ to get Eq. (17a),

$$
\rho(0)=\frac{\alpha}{1+\alpha} .
$$

This can be seen as if the left density was imposed by the reservoir density to which the system is coupled, which is not $\rho$, but $\rho /(1-\rho)$, therefore $\frac{\rho}{\rho_{s}}=\frac{\rho}{1-\rho}=\alpha$. Note that the current-density relation leads to the following boundary condition on the current:

$$
j(0)=j_{\alpha}=\frac{\alpha(1-\alpha)}{1+\alpha} .
$$

Right boundary. On the right boundary the last two sites are emptied at the same time and there is no exclusion on the last site $i=N$, therefore the incoming current on the second to last site $i=N-2$ is the usual one while the outgoing current is given by the occupation of the site $i=N-1$ (since the last site is empty); consistently with the continuity property, the outgoing current at site $i=N-2$ is also the gain term for the last site, while the loss term is given by $\beta \rho_{N}$ :

$$
\begin{gathered}
\partial_{\tau} \rho_{N-1}=\frac{\rho_{N-2}\left(1-\rho_{N-1}-\rho_{N}\right)}{1-\rho_{N-1}}-\rho_{N-1}, \\
\partial_{\tau} \rho_{N}=\rho_{N-1}-\beta \rho_{N} .
\end{gathered}
$$

It is obvious that in the continuum limit the density in the last two sites is different and only a coarse grained quantity like the average density (which is half the coverage density) makes sense: $\rho=\left(\rho_{N-1}+\rho_{N}\right) / 2$. One considers this last definition, the stationary state of Eq. (B5a) with constant incoming current [that gives $\rho(1-2 \rho) /(1-\rho)=\rho_{N-1}$ ] and the stationary state of Eq. (B5b) (that gives $\rho_{N-1}=\beta \rho_{N}$ ), to obtain

$$
\frac{\rho(1-2 \rho)}{1-\rho}=\rho \frac{2 \beta}{1-\beta},
$$

which finally gives the right boundary condition:

$$
\rho(1)=\frac{1-\beta}{2} \text {. }
$$

The right boundary condition for the current reads

$$
j(1)=j_{\beta}=\frac{\beta(1-\beta)}{1+\beta},
$$

which has naturally the same form of the left condition because of the effective particle hole symmetry.

Note also that in the HD phase the particles wait a long time before detaching giving rise to peculiar correlations: the (coverage) density profile exhibits a sawtooth profile superimposed to the analytical one. By using linear analysis on the discrete map it can be shown that these correlations decay exponentially (but not as fast as the usual boundary layers) to the fixed point. 
[1] For a review, see e.g., H. Hinsch, R. Kouyos, and E. Frey, in Traffic and Granular Flow '05, edited by A. Schadschneider, T. Pöschel, R. Kühne, M. Schreckenberg, and D. E. Wolf (Springer, Berlin, 2006).

[2] C. MacDonald, J. Gibbs, and A. Pipkin, Biopolymers 6, 1 (1968).

[3] C. MacDonald and J. Gibbs, Biopolymers 7, 707 (1969).

[4] J. Krug, Phys. Rev. Lett. 67, 1882 (1991).

[5] B. Derrida, E. Domany, and D. Mukamel, J. Stat. Phys. 69, 667 (1992)

[6] G. Schütz and E. Domany, J. Stat. Phys. 72, 277 (1993).

[7] B. Derrida, M. Evans, V. Hakim, and V. Pasquier, J. Phys. A 26, 1493 (1993).

[8] G. Schütz, Phys. Rev. E 47, 4265 (1993).

[9] J. de Gier and F. H. L. Essler, Phys. Rev. Lett. 95, 240601 (2005).

[10] M. Dudziński and G. Schütz, J. Phys. A 33, 8351 (2000).

[11] P. Pierobon, A. Parmeggiani, F. von Oppen, and E. Frey, Phys. Rev. E 72, 036123 (2005)

[12] G. Schütz, Exactly Solvable Models in Many-body Systems, Phase Transition and Critical Phenomena, Vol. 19 (Academic Press, London, 2001).

[13] B. Derrida, Phys. Rep. 301, 65 (1998).

[14] B. Schmittmann and R. Zia, Statistical Mechanics of Driven Diffusive Systems, Phase Transition and Critical Phenomena, Vol. 17 (Academic Press, London, 1995)

[15] G. Lakatos and T. Chou, J. Phys. A 36, 2027 (2003).

[16] L. B. Shaw, R. K. P. Zia, and K. H. Lee, Phys. Rev. E 68 , 021910 (2003).

[17] F. C. Alcaraz and R. Z. Bariev, Phys. Rev. E 60, 79 (1999).

[18] G. Schönherr and G. Schütz, J. Phys. A 37, 8215 (2004).

[19] B. Alberts, A. Johnson, J. Lewis, M. Raff, K. Roberts, and P. Walter, Molecular Biology of the Cell, 4th ed. (Garland Science, New York, 2002).

[20] J. Howard, Mechanics of Motor Proteins and the Cytoskeleton (Sinauer Associates, Inc., Sunderland, MA, 2001).

[21] R. Lipowsky, S. Klumpp, and T. M. Nieuwenhuizen, Phys. Rev. Lett. 87, 108101 (2001).

[22] A. Parmeggiani, T. Franosch, and E. Frey, Phys. Rev. Lett. 90, 086601 (2003).

[23] J. Howard, Mechanics of Motor Proteins and the Cytoskeleton (Ref. [20]) Chap. 13

[24] L. Tonks, Phys. Rev. 50, 955 (1936).

[25] C. Thompson, Classical Equilibrium Statistical Mechanics (Oxford University Press, New York, 1988).

[26] J. McGhee and P. von Hippel, J. Mol. Biol. 86, 469 (1974).

[27] P. Flory, J. Am. Chem. Soc. 61, 1518 (1939).

[28] J. Evans, Rev. Mod. Phys. 65, 1281 (1993).

[29] D. Mattis and M. Glasser, Rev. Mod. Phys. 70, 979 (1998).

[30] Nonequilibrium Statistical Mechanics in One Dimension, edited by V. Privman (Cambridge University Press, Cambridge, UK, 1997).

[31] V. Privman and P. Nielaba, Europhys. Lett. 18, 673 (1992).
[32] A slight modification of the dynamical rules at the boundaries, e.g., allowing particles to enter (leave) the system gradually, has been shown not to introduce new features (see Refs. $[15,16]$ for discussion).

[33] A. Bortz, M. Kalos, and J. Lebowitz, J. Chem. Phys. 17, 10 (1975).

[34] D. Landau and K. Binder, Monte Carlo Simulations in Statistical Physics (Cambridge University Press, Cambridge, UK, 2000).

[35] D. Gillespie, J. Phys. Chem. 81, 2340 (1977).

[36] E. Frey, A. Parmeggiani, and T. Franosch, Genome Inform. 15, 46 (2004).

[37] K. Nishinari, Y. Okada, A. Schadschneider, and D. Chowdhury, Phys. Rev. Lett. 95, 118101 (2005).

[38] For a linear death process the corresponding flux follows immediately from the master equation $[43,44]$

[39] This approximation has been introduced first in the context of pure $\ell$-TASEP $[2,3]$.

[40] The grand canonical partition function could be expressed in terms of a Chebyshev polynomial of the second kind $\mathcal{Z}_{N}=($ $-x)^{N / 2} U_{N}\left(\frac{1}{2 \sqrt{-x}}\right)$; Eq. (11) is merely a different representation.

[41] A. Parmeggiani, T. Franosch, and E. Frey, Phys. Rev. E 70, 046101 (2004).

[42] E. Frey and A. Vilfan, Chem. Phys. 284, 287 (2002).

[43] N. V. Kampen, Stochastic Process in Physics and Chemistry (North-Holland, Oxford, UK, 1981).

[44] C. Gardiner, Handbook of Stochastic Methods (SpringerVerlag, Heidelberg, 1985).

[45] For Eq. (31) the replacement is obvious in the condition, whereas for Eq. (30) notice that $R_{\beta}\left(\rho_{\beta}\right)$ depends on $\beta$ according to Eq. (28b).

[46] All the phase boundaries between the LD-HD and HD phases intersect at a fixed nodal point. At this point the solution is independent of $\Omega_{D}$ but depends on the binding constant and on the boundaries: this is the point where the bulk density imposed by the right boundary becomes equal to the isotherm, moreover, since it is valid also in the limit of small $\Omega_{D}$ one expects $\alpha=\beta$. The coordinates of such a point $\left(\alpha_{N}, \beta_{N}\right)$ can be computed from $\rho_{\beta}(1)=(1-\beta) / 2=\rho_{I}$ and are equal to $\alpha=\beta$ $=1 / \sqrt{4 K+1}$

[47] In this case too there is a nodal point where all the phase boundaries between coexistence and HD (or $M$ ) phase intersect: it can be computed from the condition $\rho_{\alpha}(0)=\alpha /(1+\alpha)$ $=\rho_{I}$ and corresponds to the point $\alpha=\beta=(\sqrt{4 K+1}$ $-1) /(\sqrt{4 K+1}+1)$.

[48] This is not always the case: systems where interactions between particles are taken into account, as in Refs. [49,50], showed current-density relation with multiple maxima.

[49] J. S. Hager, J. Krug, V. Popkov, and G. M. Schütz, Phys. Rev. E 63, 056110 (2001).

[50] V. Popkov, A. Rákos, R. D. Willmann, A. B. Kolomeisky, and G. M. Schütz, Phys. Rev. E 67, 066117 (2003). 